\title{
Façonner l'avenir de la pandémie de COVID-19 au Canada
}

\author{
Ashleigh R. Tuite PhD MSP, Amy L. Greer PhD MSc
}

- Citation: CMAJ 2020 September 14;192:E1074-5. doi : 10.1503/cmaj.201758-f; diffusion hâtive le 10 août 2020

Voir la version anglaise de l'article ici : www.cmaj.ca/lookup/doi/10.1503/cmaj.201758; voir la recherche connexe en anglais ici : www. cmaj.ca/lookup/doi/10.1503/cmaj.200990; voir la recherche connexe en français ici : www.cmaj.ca/lookup/doi/10.1503/cmaj.200990-f

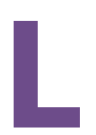

e Canada est en période d'allègement des mesures de santé publique qu'il a instaurées afin de répondre rapidement et énergiquement à la pandémie de maladie à coronavirus 2019 (COVID-19). Plusieurs autres pays font de même. Pour déterminer le meilleur chemin à suivre, il faut à présent prendre une série de décisions délicates à plusieurs points de vue, comparativement à la phase initiale de la pandémie. Le temps est venu de définir l'avenir que nous souhaitons et de travailler à l'atteindre.

Une étude de modélisation mathématique connexe, par $\mathrm{Ng}$ et ses collègues ${ }^{1}$, analyse les trajectoires que pourrait prendre l'épidémie de COVID-19 dans la population canadienne selon divers scénarios d'interventions non pharmaceutiques. D'après ses conclusions, notre avenir est très incertain et il dépend de nos choix. Avec la multiplication des tests de dépistage du coronavirus du syndrome respiratoire aigu sévère 2 (SRAS-CoV-2), une recherche rapide des contacts, l'isolement sans délai des personnes infectées, la distanciation physique et la fermeture des endroits publics, $\mathrm{Ng}$ et ses collègues. projettent des taux d'attaque négligeables dans la population $(<1 \%$ de la population contracterait l'infection); par contre, avec l'érosion de ces mesures, jusqu'à $56 \%$ de la population canadienne pourrait contracter le SRAS-CoV-2. Étant donné un tel éventail de possibilités, comment s'assurer de suivre la voie qui limitera la transmission du virus et préviendra une issue négative? Et, compte tenu que le SRAS-CoV-2 continuera de circuler dans la population canadienne dans un avenir prévisible, comment maîtriser la transmission à long terme tout en limitant les graves répercussions sociales et économiques?

La modélisation mathématique peut guider le choix des décideurs pour ce qui est des mesures à prendre pour maîtriser l'épidémie. Comme le montrent $\mathrm{Ng}$ et ses collègues ${ }^{1}$, les combinaisons d'interventions ont de meilleures chances d'être plus efficaces que les mesures de santé publique isolées, particulièrement dans un contexte où les collectivités ne sont plus fortement confinées et où les écoles et les milieux de travail ouvrent. Mais l'efficacité de différentes interventions à réduire la transmission n'est qu'un morceau du casse-tête. L'adhésion de la population à

\section{POINTS CLÉS}

- L'avenir de la pandémie de maladie à coronavirus 2019 au Canada est incertain et dépend fortement des gestes posés aujourd'hui.

- De multiples interventions en santé publique seront probablement nécessaires dans un avenir prévisible pour prévenir la transmission incontrôlée du coronavirus du syndrome respiratoire aigu sévère 2 (SRAS-CoV-2).

- Les modèles mathématiques peuvent aider à comprendre quelles combinaisons de mesures de santé publique sont requises pour maîtriser l'épidémie alors que le Canada lève ses restrictions, mais pour déterminer de quelle façon procéder au déconfinement, il faut tenir compte de l'influence d'autres facteurs, de même que des priorités et des préférences de la société.

- II ne sera peut-être pas possible d'éliminer le SRAS-CoV-2 dans le contexte canadien, mais l'objectif n'en demeure pas moins louable.

ces mesures est aussi un facteur important, particulièrement à un moment où la situation semble s'alléger, la menace se fait moins présente et la «fatigue de la pandémie » s'installe. En l'absence d'un vaccin ou d'un traitement, le chemin sera long. Le modèle de $\mathrm{Ng}$ et ses collègues s'étale sur un horizon temporel prolongé (janvier 2022), et même les quelques scénarios qui parlent d'une élimination de la COVID-19 n'y arrivent qu'après un déploiement des mesures pendant plus d'une année'.

Il n'y a pas une voie universelle à suivre pour affronter cette pandémie. Les décideurs doivent déterminer de quelle façon utiliser stratégiquement les mesures de santé publique afin de maîtriser l'épidémie le mieux possible et voir si certains aspects de la réponse initiale à la pandémie ne méritent pas qu'on y investisse des ressources additionnelles. Ultimement, aucun choix ne sera universellement bon pour tous, c'est pourquoi qu'il faut prioriser avec grand soins les dimensions à privilégier aux plans social et économique. Walsh a proposé aux décideurs de réfléchir aux dimensions de la vie sociale à déconfiner en regardant par la lorgnette $d u$ « budget des interactions sociales $»^{2}$. Chaque interaction 
sociale comporte un risque de transmission du SRAS-CoV-2 proportionnel au volume des groupes, à l'étroitesse des contacts et à leur tenue à l'intérieur ${ }^{3}$. Seul un budget des interactions sociales limité peut être dépensé avant que la transmission de la COVID-19 n'augmente substantiellement. L'épuisement de ce budget forcera la reprise des fermetures agressives. Par exemple, si on priorise la réouverture des écoles et la réunification des familles et des aidants auprès des résidents de centres d'hébergement de soins de longue durée, il faudra peut-être renoncer à la réouverture des espaces intérieurs des bars et des restaurants, puisque ensemble, ces activités font substantiellement augmenter le risque de transmission du SRAS-CoV-2. De même, le retour en classe des élèves dans les conditions les plus sûres et inclusives possibles pourrait forcer l'annulation d'activités plus à risque dans les écoles, telles que réunions générales et chorales ${ }^{4}$.

Il s'agit de décisions complexes, coûteuses et de nature foncièrement politique. Elles auront des conséquences importantes (et imprévisibles). Elles ont le potentiel d'améliorer ou d'apaiser les inégalités sanitaires et économiques actuelles ${ }^{5}$, et elles doivent, en outre, être prises dans un contexte d'incertitude. Les modèles de transmission du SRAS-CoV-2 peuvent aider à clarifier certaines prémisses sous-jacentes et à établir les priorités, en plus de permettre aux décideurs de faire des choix à partir des meilleures données disponibles. Par contre, la plupart des modèles ne tiennent compte que des dimensions sanitaires et considèrent rarement les retombées sociales et économiques indirectes des différents choix.

Le budget des interactions sociales au Canada n'est pas fixe; toutefois, il peut augmenter avec des interventions plus efficaces. Par exemple, une récente étude de modélisation mathématique a montré l'importance de tester à grande échelle les cas de COVID-19 symptomatiques et de procéder rapidement à la recherche des contacts ${ }^{6}$. Investir davantage dans les capacités de dépistage et obtenir les résultats des tests plus rapidement de manière à amorcer la recherche de contacts permettraient l'élargissement du budget d'interactions sociales. Il faut s'inspirer des pays qui ont maîtrisé l'épidémie de COVID-19 avec succès. Par exemple, l'approche japonaise ${ }^{3}$, qui consiste à repérer rapidement les groupes de cas et à dégager leurs caractéristiques communes, pourrait être utilisée là où des tests à grande échelle semblent irréalisables.

$\mathrm{Ng}$ et ses collègues montrent aussi qu'il est peu probable qu'on arrive à éliminer le SRAS-CoV-2 sans mesures restrictives ${ }^{1}$. En santé publique, on parle d'« élimination » quand on arrive à ramener à zéro l'incidence de l'infection causée par un agent pathogène spécifique dans une région géographique donnée ${ }^{7}$. Dans la mesure où la transmission persiste à l'extérieur du territoire qui vise à éliminer l'infection, le risque de réintroduction demeure. C'est pourquoi on peut remettre en question l'objectif d'éliminer l'infection dans un pays comme le Canada, où la connectivité globale est grande. Même si l'élimination est irréaliste, nous pourrions néanmoins trouver qu'il s'agit d'un objectif louable.
L'élimination devrait être le but et la principale préoccupation au Canada. En misant sur un leadership fort, une grande ingéniosité, le sens de l'organisation et de solides mesures de santé publique, le Canada pourra repérer les cas importés et limiter la transmission communautaire, ce qui facilitera la réouverture de la société et le retour à un sentiment de normalité. En effet, une meilleure maîtrise de l'épidémie de COVID-19 semble être la clé pour minimiser les répercussions économiques négatives ${ }^{8}$.

On comprend beaucoup mieux le SRAS-CoV-2 et la COVID-19 maintenant que lorsque le virus a fait son apparition. Certaines interventions de la santé publique ont permis de maîtriser efficacement la transmission. Les gestes que nous posons et les politiques que nous mettons en place façonneront l'avenir de cette pandémie.

\section{Références}

1. Ng V, Fazil A, Waddell LA, et al. Projected effects of nonpharmaceutical public health interventions to prevent resurgence of SARS-CoV-2 transmission in Canada. CMAJ 10 août 2020 [Cyberpublication avant impression]. doi: 10.1503/ cmaj.200990.

2. Walsh S. We are asking the wrong questions about easing lockdown [blog]. The BMJ Opinion 2 juin 2020. Londres (Royaume-Uni). The BMJ. [En ligne]. Accessible ici : https://blogs.bmj.com/bmj/2020/06/02/sebastian-walsh-we-are-asking-the -wrong-questions-about-easing-lockdown/ (consulté le 17 juillet 2020).

3. Furuse Y, Sando E, Tsuchiya N, et al. Clusters of coronavirus disease in communities, Japan, January-April 2020. Emerg Infect Dis 10 août 2020 [Cyberpublication avant impression]. doi 10.3201/eid2609.202272.

4. Hamner L, Dubbel P, Capron I, et al. High SARS-CoV-2 attack rate following exposure at a choir practice-Skagit County, Washington, March 2020. MMWR Morb Mortal Wkly Rep 2020;69:606-10.

5. Ali S, Asaria M, Stranges S. COVID-19 and inequality: Are we all in this together? Can J Public Health 2020;111: 415-6.

6. Kucharski AJ, Klepac P, Conlan AJK, et al.; CMMID COVID-19 working group. Effectiveness of isolation, testing, contact tracing, and physical distancing on reducing transmission of SARS-CoV-2 in different settings: a mathematical modelling study. Lancet Infect Dis 15 juin 2020 [Cyberpublication avant impression]. doi: 10.1016/S1473-3099(20)30457-6.

7. Dowdle WR. The principles of disease elimination and eradication. Bull World Health Organ 1998;76 (Suppl 2):22-5.

8. Aum S, Lee SY, Shin Y. COVID-19 doesn't need lockdowns to destroy jobs: the effects of local outbreaks in Korea. SSRN 3 juin 2020. Accessible ici : https:// ssrn.com/abstract=3615585 (consulté le 17 juillet 2020).

\section{Intérêts concurrents : Aucun déclaré.}

Cet article a été sollicité et il n'a pas été révisé par des pairs.

Affiliations : École Dalla Lana de santé publique (Tuite), Université de Toronto, Toronto, Ont.; Département de médecine des populations (Greer), Université de Guelph, Guelph, Ont.

Collaborateurs : Les deux auteurs ont contribué à la conception du travail, ont rédigé le manuscrit et en ont révisé de façon critique le contenu intellectuel important; ils ont donné leur approbation finale pour la version destinée à être publiée et assument l'entière responsabilité de tous les aspects du travail.

Correspondance : Ashleigh Tuite, ashleigh.tuite@utoronto.ca 